\title{
Elevated Expression of Hypoxanthine Guanine Phosphoribosyltransferase within Malignant Tissue
}

\author{
Michelle H. Townsend ${ }^{1}$, Abigail M. Felsted ${ }^{1}$, Zachary E. Ence ${ }^{2}$, Stephen R. Piccolo ${ }^{2,3}$, Richard A. Robison ${ }^{1}$ \& \\ Kim L. O'Neill ${ }^{1}$ \\ ${ }^{1}$ Department of Microbiology and Molecular Biology, Brigham Young University, Provo UT 84602, USA \\ ${ }^{2}$ Department of Biology, Brigham Young University, Provo UT 84602, USA \\ ${ }^{3}$ Department of Biomedical Informatics, University of Utah, Salt Lake City UT 84132, USA \\ Correspondence: Kim L. O'Neill, 3142 LSB, Brigham Young University, Provo, UT 84602, USA. Tel: \\ 801-422-2449. E-mail: kim_oneill@byu.edu
}

Received: July 28, $2017 \quad$ Accepted: August 23, $2017 \quad$ Online Published: September 13, 2017

doi:10.5539/cco.v6n2p19 URL: http://dx.doi.org/10.5539/cco.v6n2p19

\begin{abstract}
Hypoxanthine Guanine Phosphoribosyltransferase (HPRT) is a housekeeping enzyme involved in the purine synthesis of guanine and inosine in the salvage pathway. While other salvage pathway enzymes, such as TK1, have been established as biomarkers for both cancer cell proliferation and cancer development, little research been done to evaluate whether HPRT has the same potential as a cancer biomarker. We designed this study to determine if HPRT has value as an identifier of malignancy within the most common types of cancer. We utilized histological samples from lung, colon, prostate, and breast cancer with additional normal tissue to evaluate whether there was any elevation of HPRT within malignant samples. In addition, we also assessed general HPRT expression within patient's samples from The Cancer Genome Atlas (TCGA) to confirm clinical relevance. We found that within a subset of patients, there was significant elevation of HPRT when compared to normal tissue controls. This elevation was seen in 33-55\% of the malignant samples and appears to have no dependence on cancer stage. There were slight differences in staining patterns among all the organ types, but overall each organ displayed the same pattern of 'HPRT high' and 'HPRT low' populations within malignant samples. We found that in our TCGA samples, there was a similar elevation of HPRT that was significant when compared to normal controls. Overall, as an upregulated enzyme that does not directly correlate with stage, HPRT could become a valuable marker in the early diagnosis of a variety of solid tumors.
\end{abstract}

Keywords: breast cancer, colon cancer, Hypoxanthine Guanine Phosphoribosyltransferase (HPRT or HGPRT), lung cancer, prostate cancer

\section{Introduction}

With 14 million new cases diagnosed and 8.2 million deaths reported worldwide in 2012, cancer is a leading global health concern ("Worldwide cancer statistics | Cancer Research UK."). In 2016, the most common malignancies reported were lung, breast, prostate, and colon which comprised approximately $44.5 \%$ (Lung 224,390; Breast - 249,260; Prostate - 180,890; Colon - 95,270) of all new cancer cases in the United States (American Cancer Society, 2016; R. L. Siegel, Miller, \& Jemal, 2016; R. Siegel, Miller, \& Jemal, 2015). As the most common cancers throughout the world, new biomarkers are constantly needed to identify cancer in early stages to decrease mortality. While several cancer markers have been identified for each of these diseases, Hypoxanthine Guanine Phosphoribosyltransferase (HPRT) has the potential to provide an additional diagnostic tool for several cancer types.

HPRT is a transferase responsible for the salvage of both guanine and inosine nucleotides throughout the cell cycle (Keough, Brereton, De Jersey, \& Guddat, 2005; Monnat, Chiaverotti, Hackmann, \& Maresh, 1992; Stout \& Caskey, 1985; Wilson, Tarr, \& Kelley, 1983). As an established human reporter gene, HPRT is currently utilized to provide understanding of somatic mutations and mutagenesis in both in vitro and in vivo systems (Chang, Tseng, Lin, Chuang, \& Chao, 2017; Gobrecht, McDyre, Comotto, \& Reynolds, 2017; Grist, McCarron, Kutlaca, Turner, \& Morley, 1992; Hirota, Kubota, Hashimoto, Adachi, \& Mikawa, 1993; O’Neill et al., 1994). Mutation events in this locus are extensively monitored in population studies to evaluate the effects of continuing exposure 
to mutagens and detect carcinogenic agents that lead to increased cancer risk (Albertini, 2001; Hou et al., 1999; Vacek \& Albertini, 1993). In at-risk populations including smokers, patients with DNA repair deficiency syndromes, and atom bomb survivors there are significant mutations in the HPRT locus that directly corresponds with higher cancer incidence (Branda, O'Neill, Jacobson-Kram, \& Albertini, 1992; Cheng et al., 1995; Duthie, Ross, \& Collins, 1995; Hakoda, Akiyama, Kyoizumi, \& Awa, 1988; Hou et al., 1999; Robinson et al., 1994; Sawada et al., 1998; Tates, Dam, Natarajan, Zwinderman, \& Osanto, 1994). While its role as a standard mutational biomarker for cancer development has been well established, the relevance of HPRT to the proliferative capacity and tumorigenesis of cancer has not been evaluated. It has been established that other salvage enzymes, such as TK1, have a direct relevance to cancer stage and aggressiveness as serum detection of the enzyme is correlated to cancer stage and recurrence (Alegre et al., 2014; Alegre, Robison, \& O'Neill, 2012; Alegre, Robison, \& O’Neill, 2013; He et al., 2006; Jagarlamudi, Hansson, \& Eriksson, 2015). Additionally, recent evidence has also shown that HPRT may play a unique role in cancer as it is presented on the surface of certain cancer cells (Townsend et al., 2017). To address whether the same stage-dependent protein elevation pattern existed for HPRT as with TK1, and to assess its use as a useful biomarker in clinical samples we have evaluated the expression of the enzyme in hundreds of patient samples. In addition, to determine if HPRT could serve as a cancer biomarker for early cancer detection, we compare HPRT expression in the most commonly diagnosed cancers throughout the world (lung, breast, prostate, and colon).

\section{Methods}

\subsection{Chemicals/Reagents}

DIVA Decloaker 10x, Background Sniper, Mach 4 HRP polymer, DAB Peroxidase, Hematoxylin, Hydrophobic pen, and Universal Negative antibodies were all obtained from Biocare Medical, Concord, CA. Anti-HPRT monoclonal antibody (Abcam, Cambridge, UK) was aliquoted and stored at $-20^{\circ} \mathrm{C}$. GAPDH polyclonal antibody (One World Labs, San Diego CA) was aliquoted and stored at $-20^{\circ} \mathrm{C}$. Tween20 (Fisher Reagents, Waltham MA) was stored at room temperature. 30\% Hydrogen Peroxide (Fisher Reagents, Waltham MA) was stored at $4{ }^{\circ} \mathrm{C}$.

\subsection{Patient Samples}

All tissue microarrays were obtained from Biomax and stained for HPRT, GAPDH, and an isotype antibody to evaluate protein expression and upregulation. Lung samples were evaluated from 54 patients ranging in age from 39-77 containing malignant $(\mathrm{n}=17)$, normal $(\mathrm{n}=18)$, and marginal tissue $(\mathrm{n}=17)$ samples. Malignant tissue ranged from grade 1-3 and included female $(n=4)$ and male $(n=13)$ patients with either large cell carcinoma $(n=3)$, adenocarcinoma $(n=5)$, or squamous carcinoma $(n=6)$. Colon samples were evaluated from 100 patients ranging in age from 30-79 with colon adenocarcinoma $(n=30)$, metastatic adenocarcinoma from the colon $(n=30)$, tubular adenoma $(n=10)$, cancer adjacent normal tissue $(n=20)$, and normal colon tissue $(n=10)$. Sex (male, $n=63$; female, $\mathrm{n}=37$ ) and grade (1-3) were variable between samples. Breast samples were analyzed from 63 patients ranging in age from 29-68 containing malignant $(n=18)$, normal $(n=24)$, and margin of carcinoma samples $(n=21)$. Prostate samples were analyzed from 63 patients ranging in age from 60-87 containing adenocarcinoma $(n=60)$ and hyperplasia $(\mathrm{n}=3)$ samples.

\subsection{Immunohistochemistry}

HPRT levels were assessed using standard immunohistochemistry staining. Tissues were treated with Histoclear (National Diagnostics, Charlotte, North Carolina) and subsequently rehydrated in a series of ethanol washes before treatment with a DIVA Decloaker solution to retrieve antigens. Tissues were washed with a diluted hydrogen peroxide solution followed by a Tris Buffered Saline-Tween20 (TBST) wash. Following washing, tissues were incubated with a blocking Background Sniper solution to reduce non-specific antibody binding. Following blocking, a primary antibody was added to the tissue at a dilution of 1:100 and incubated overnight at $4^{\circ} \mathrm{C}$. Tissues were then washed and then treated with secondary antibody conjugated to a HRP polymer and incubated for an hour. Following washing, a DAB Peroxidase solution was incubated with the tissues. Areas of antibody binding converted the colorless substrate to a brown product, effectively highlighting the target protein. Tissues were subsequently stained with hematoxylin to stain the nucleus of the cells. Along with HPRT treatment, a universal negative antibody was used as a negative control, and a GAPDH antibody was used as a positive control.

\subsection{Tissue Quantification}

Tissues were quantified utilizing ImageJ software. All images were evaluated using the IHC toolbox ImageJ plugin. The DAB option was chosen and the tissue image was removed of all other staining. Following this modification, the image is then converted to a grayscale and a threshold was applied in order to eliminate areas 
of negative space inherit within the tissue image. Once a universal threshold was applied, the average gray value of the tissue was collected. The same threshold was applied to all tissue samples within the same organ in order to ensure consistency and reduce bias.

\subsection{Bioinformatic Analysis}

We evaluated differences in expression levels of the HPRT gene in 3,147 tumor and 316 normal samples from The Cancer Genome Atlas (TCGA). RNA-sequencing data that had been processed using the featureCounts algorithm to transcripts-per-million values was utilized. The normal expression data were from adjacent normal tissue or blood samples and were not necessarily matched to the tumor data on a per-sample basis. We parsed and prepared the data using the Python ("Welcome to Python.org," n.d.) (v3.6.1) programming language. In making graphs, we used the R (v3.2.2) statistical software and the ggplot2 package (v.2.2.1).

\subsection{Statistical Analysis}

Comparison between tissue samples was conducted utilizing ANOVA statistical analysis with the multiple comparison method. Unpaired $t$ tests were utilized in conjunction to confirm statistical significance. Statistical analyses were evaluated using GraphPad Prism 7 software. Differences were considered significant when the $p$ value was $<0.05$. Additionally, when comparing RNA expression values between normal and malignant samples in TCGA, we used the Mann-Whitney U test with an exact p-value calculation. These analyses were completed using the R statistical software. Asterisks were used in figures to indicate levels of significance with $\mathrm{ns}=\mathrm{P}>0.5$, $*=\mathrm{P} \leq 0.05, * *=\mathrm{P} \leq 0.01, * * *=\mathrm{P} \leq 0.001$, and $* * * *=\mathrm{P} \leq 0.0001$.

\section{Results}

3.1 HPRT has Variable Expression within Several Cancers and Shows an overall Upregulation in Malignant

\section{Tissue}

Within all malignant tissue there were populations of patients with high HPRT expression and populations with relatively low HPRT expression. We have labeled these patients as "HPRT high" and "HPRT low" (Figure 1). Patients with high levels (Figure 1A) were significantly separated from patients with low levels (Figure 1B), which had staining characteristics similar to normal tissue (Figure 1C). The variability of HPRT within malignant tissue was also variable between cancer types as each organ had a different percentage of patients who experienced an upregulation (Lung-33\%, Breast-55\%, Colon-33\%, Prostate-47\%). These data indicate that HPRT is only elevated within some patients, and may serve as a diagnostic marker for characterizing tumors. 


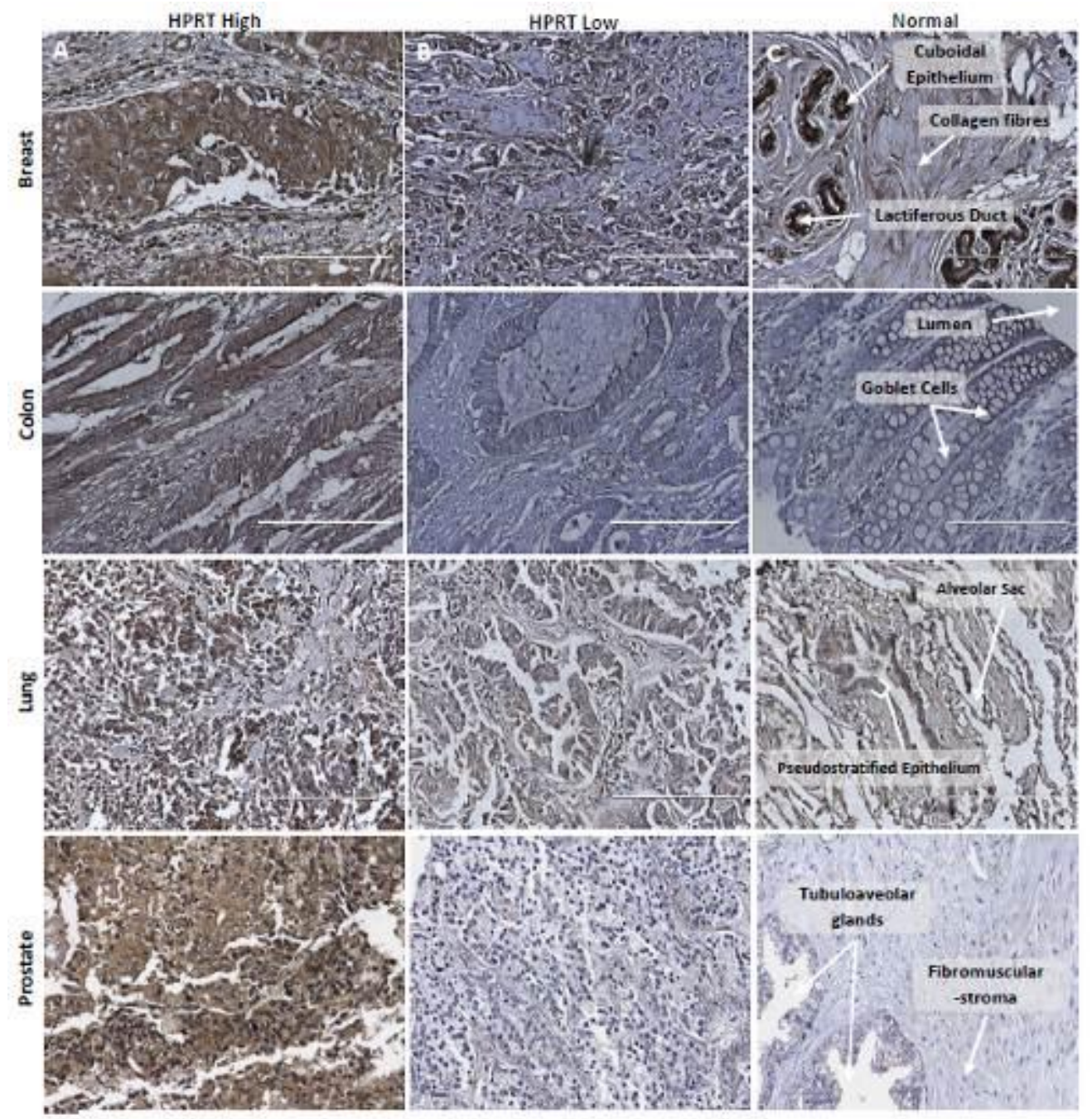

Figure 1. HPRT staining within malignant and normal breast, colon, lung, and prostate

A) All organs observed contained a population of patients who had a significant increase in HPRT staining, which were labeled "HPRT High". These tissues were significant when compared to normal controls. B) Organs also contained a population of patients who did not experience an increase in HPRT when compared to normal controls and were labeled "HPRT Low". C) Normal tissue was stained within all organs to provide a standard to compare expression. Normal tissue is labeled with identifying features.

While a majority of staining was limited to malignant tissue, there were instances within normal lung and breast tissue where there was significant HPRT staining (Table 1). As HPRT is a housekeeping gene present within all somatic tissue, we expected to have a basal level of staining within normal tissue, and all analyses were performed against normal tissue staining to highlight any upregulation. Upon further analysis with protein expression data from clinical samples in TCGA, we found that there was a significant overall upregulation of HPRT within all cancer types evaluated when compared to normal controls (Figure 2). Samples from 1119 breast invasive carcinoma $\left(p=1.66 \times 10^{-42}\right), 483$ colon adenocarcinoma $\left(p=9 \times 10^{-18}\right), 541$ lung adenocarcinoma $(p=$ $\left.3.16 \times 10^{-32}\right), 502$ lung squamous carcinoma $\left(p=1.49 \times 10^{-59}\right)$, and 502 prostate adenocarcinoma $\left(p=1.53 \times 10^{-4}\right)$ patients were compared to normal individuals and showed significant shifts in the expression of HPRT in malignant tumors, with lung samples showing the most statistically significant shift. Within each of the cancer types evaluated we observed the same pattern of 'HPRT high' and 'HPRT low' populations in the patient cohorts. Several patients with malignancy had expression levels of HPRT comparable to normal samples, however, there was a population of patients that had elevated HPRT beyond that of normal tissue staining. These results indicate that there is a subset of patients that experience unusually high levels of HPRT expression which could be used to further characterize tumors and provide a means for early detection of malignancy. 
Table 1. HPRT staining in malignant and normal tissue

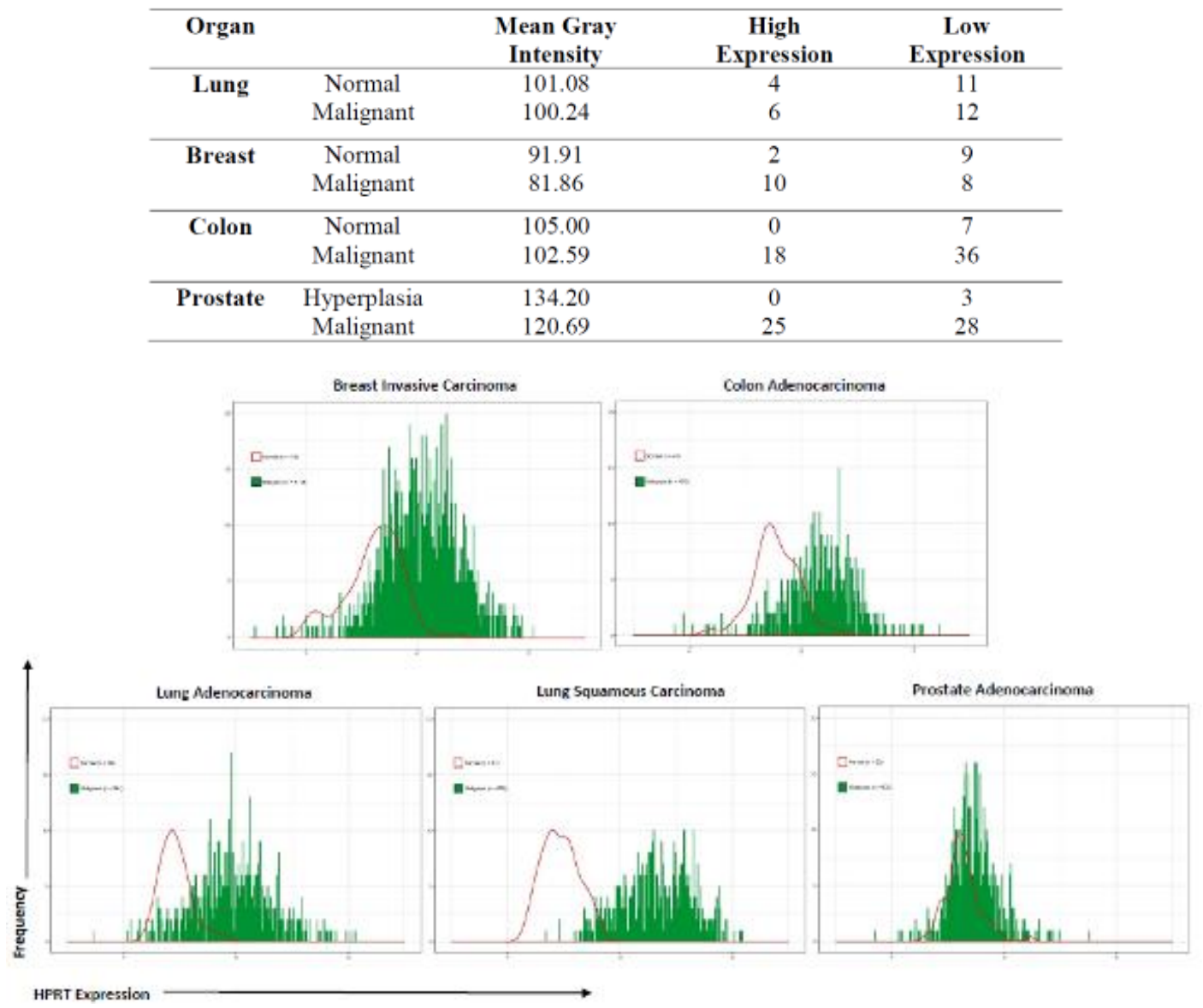

Figure 2. Expression of HPRT within TCGA tumor and normal samples

Clinical samples from 3,174 patients with various cancer types were evaluated for HPRT elevation in malignant samples when compared to normal tissue. All cancer evaluated showed a significant shift in the population of malignant samples (green) when compared to normal controls (red).

\subsection{Evaluation of HPRT within Breast Cancer Tissue Demonstrates its Potential as a Biomarker for Malignancy}

Of the 18 malignant breast tissues evaluated, 10 patients experienced a significant $(p=0.0025)$ increase in HPRT expression with an average total gray intensity of 76.32 when compared to normal breast tissue, which had an average gray intensity of 91.91 (Table 2). Normal and malignant tissue stained for HPRT were significantly different when compared to GAPDH positive and isotype negative controls (Figure 3A). In addition to normal breast tissues, adenosis, fiber tissue, and hyperplasia were evaluated and showed insignificant upregulation compared to normal samples (Figure 3B \& 3D). As a majority of the malignant tissue experienced an upregulation of HPRT, there was still a significant $(p=0.0026)$ difference between normal tissue and malignant samples when 'HPRT low' tissue was included within the analysis (Figure 3C).

Table 2. Distribution of HPRT staining in malignant breast tissue and normal breast tissue.

\begin{tabular}{lccc}
\hline Tissue Type & Number of Patients & Age Range & Overall Gray Intensity \\
\hline Adenocarcinoma High & 10 & $29-68$ & 76.32 \\
\hline Adenocarcinoma Low & 8 & $29-61$ & 88.79 \\
\hline Normal Breast Tissue & 11 & $43-69$ & 91.91 \\
\hline Hyperplasia & 3 & $49-68$ & 100.87 \\
\hline Adenosis & 7 & $28-61$ & 89.91 \\
\hline Collagen Fiber Tissue & 3 & $47-49$ & 97.30 \\
\hline Marginal Tissue & 21 & $32-74$ & 90.07 \\
\hline
\end{tabular}




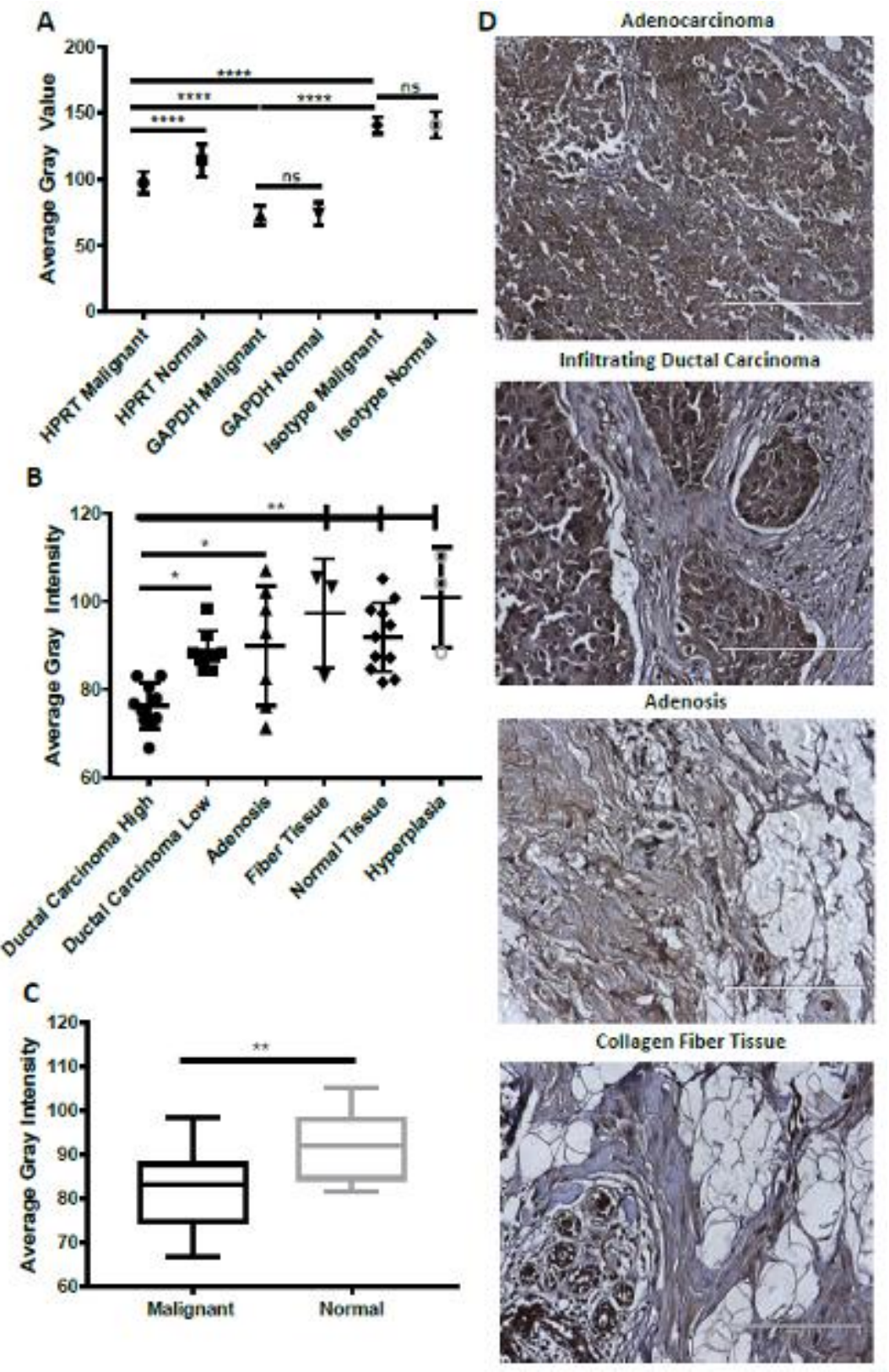

Figure 3. Statistical evaluation of breast tissue

A) Malignant and normal breast HPRT staining compared to GAPDH and Isotype controls. Expression of GAPDH and isotype staining did not have any statistically significant difference between the malignant and normal tissue. HPRT samples had a significant increase in expression when comparing malignant tissue to normal tissue. B) HPRT expression analysis between various tissue types. There were 10 patients who had significant HPRT elevation and are labeled 'Ductal Carcinoma High', while the remainder 8 patients are labeled 'Ductal Carcinoma Low' as they had staining similar to normal controls. C) Overall HPRT staining results of malignant and normal tissue within all samples. D) Tissue images of HPRT staining in various breast tissue samples.

In addition to evaluating malignant tissue, marginal tissue was analyzed to determine whether HPRT could be used to indicate unusual cell proliferation around the tumor. This analysis revealed that there was a distribution within the marginal tissue with regards to HPRT expression. We found that 6 marginal tissues were 'HPRT high' and 10 samples were 'HPRT low' (Figure 4). Each tissue showed variability, and demonstrates the ability of HPRT to aid in distinguishing potentially malignant tissue. Marginal tissue elevated in HPRT expression may indicate signs of malignancy and proliferative capacity. 

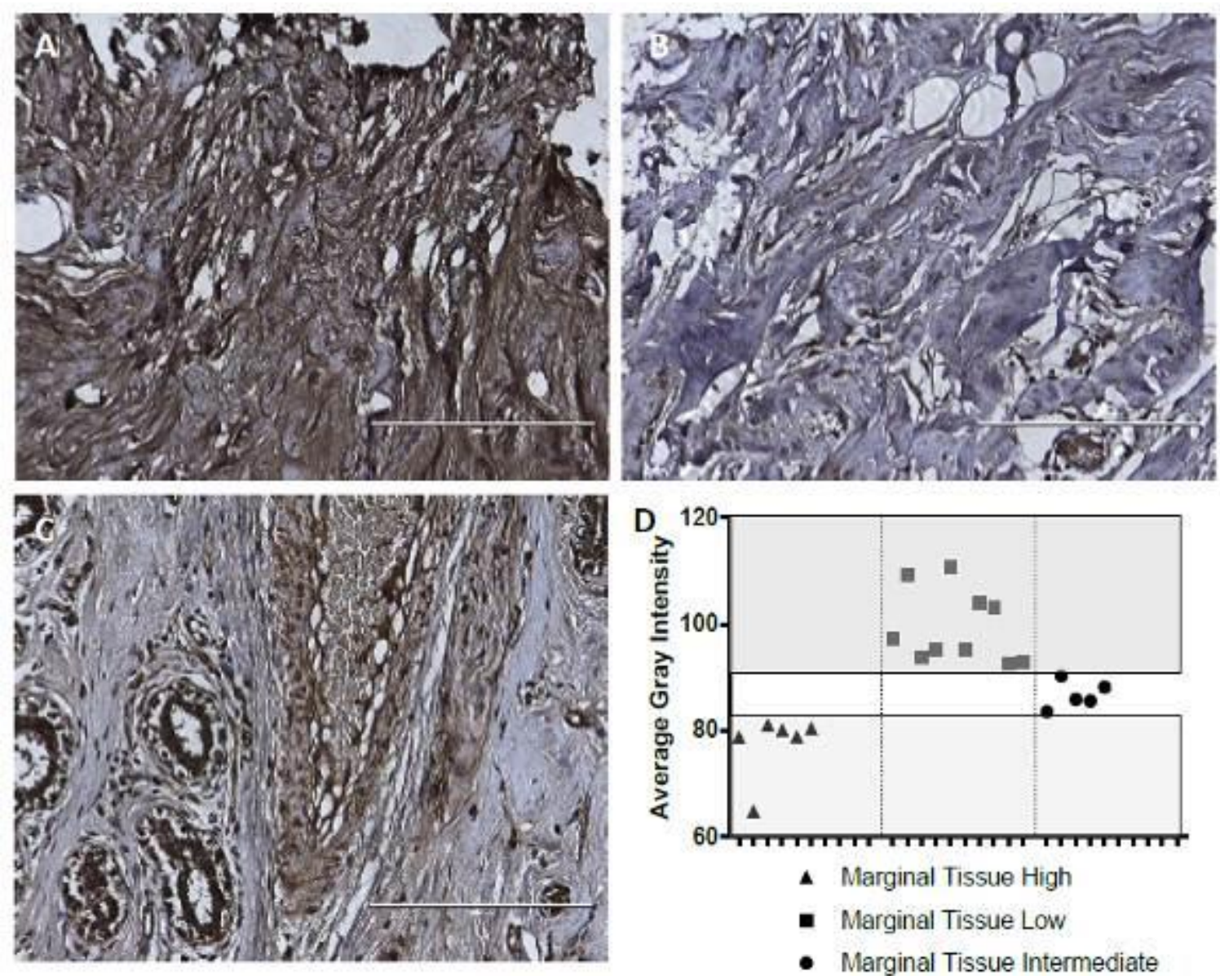

Figure 4. HPRT analysis of margin of carcinoma tissue

Margin of breast carcinoma tissue that stains A) 'HPRT High'; B) 'HPRT low'; and C) 'HPRT intermediate' staining. D) Graphical representation of the quantity of patient samples within each HPRT staining level.

Interestingly, we found that in normal tissue HPRT expression was localized to the ductal tissue. There was clear staining within the inner lining of the lactiferous ducts of the breast with minimal staining in other portions of the tissue (Figure 5). This expression is localized and may indicate HPRT involvement in cell proliferation. This same pattern of high expression in ductal openings was observed in malignant tissue, but upon HPRT staining, the cancer cells within the tumor mass all had elevated expression in "HPRT high" patients. 

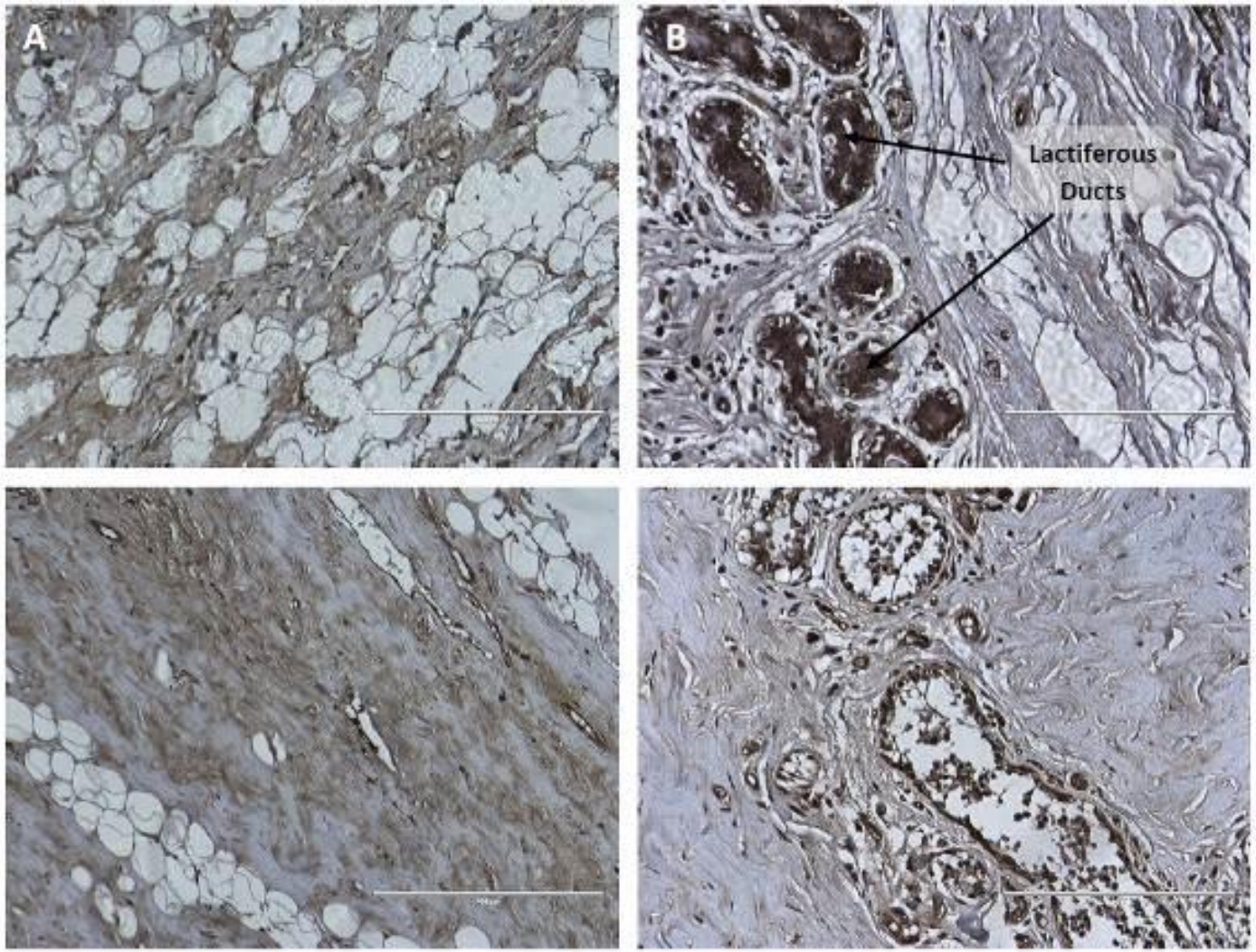

Figure 5. Normal breast tissue stained for HPRT

A) Minimal HPRT staining in normal tissue. B) While normal tissue showed no significant HPRT expression, ductal openings of normal breast tissue had unusually high staining. This may point to the involvement of HPRT in cellular proliferation.

\subsection{Lung Cancer Shows Insignificant Variability of HPRT Expression between Cancer Types and Stage}

Multiple different lung malignancies were evaluated to determine if there were any differences between the cancer types as they have significantly different origins within the lung itself. All samples were evaluated against corresponding normal, isotype controls, and GAPDH positive controls to compare expression (Figure 6A). We found that there was no statistically relevant difference between the types of malignant lung samples (squamous cell carcinoma, large cell carcinoma, adenocarcinoma, etc.) with regards to HPRT expression (Figure 6B). Adenosquamous samples had the highest average HPRT expression with a mean gray intensity of 94.12, while large cell carcinoma samples had the lowest average HPRT expression with a mean gray intensity of 104.24 (Table 3 \& Figure 3D). These values were not statistically significant and indicate that there was no differences in HPRT expression between the cancer types. Within malignant samples $33 \%$ of patients experienced significant upregulation when compared to normal tissue controls as 6 patients had expression characterized as 'HPRT High' (Figure 6C).

Table 3. Distribution of HPRT staining in malignant lung tissue and normal lung tissue

\begin{tabular}{lccccc}
\hline Tissue Type & Number of Patients & Grade Range & Age Range & M/F & Overall Gray Intensity \\
\hline Squamous Carcinoma & 3 & $2-3$ & $46-58$ & $3 / 0$ & 101.68 \\
\hline Large Cell Carcinoma & 3 & $2-3$ & $30-66$ & $3 / 0$ & 104.24 \\
\hline Adenocarcinoma & 6 & $1-3$ & $46-77$ & $5 / 1$ & 102.56 \\
\hline Alveolar Carcinoma & 2 & NA & $39-59$ & $1 / 1$ & 96.26 \\
\hline Adenosquamous Carcinoma & 2 & 3 & $60-69$ & $2 / 0$ & 94.12 \\
\hline Other Carcinomas & 2 & 3 & $59-69$ & $0 / 2$ & 95.45 \\
\hline Normal Lung Tissue & 18 & - & $39-77$ & $14 / 4$ & 101.08 \\
\hline Marginal Tissue & 18 & - & $30-77$ & $14 / 4$ & 100.74 \\
\hline
\end{tabular}

M/F; Male/Female patients. 


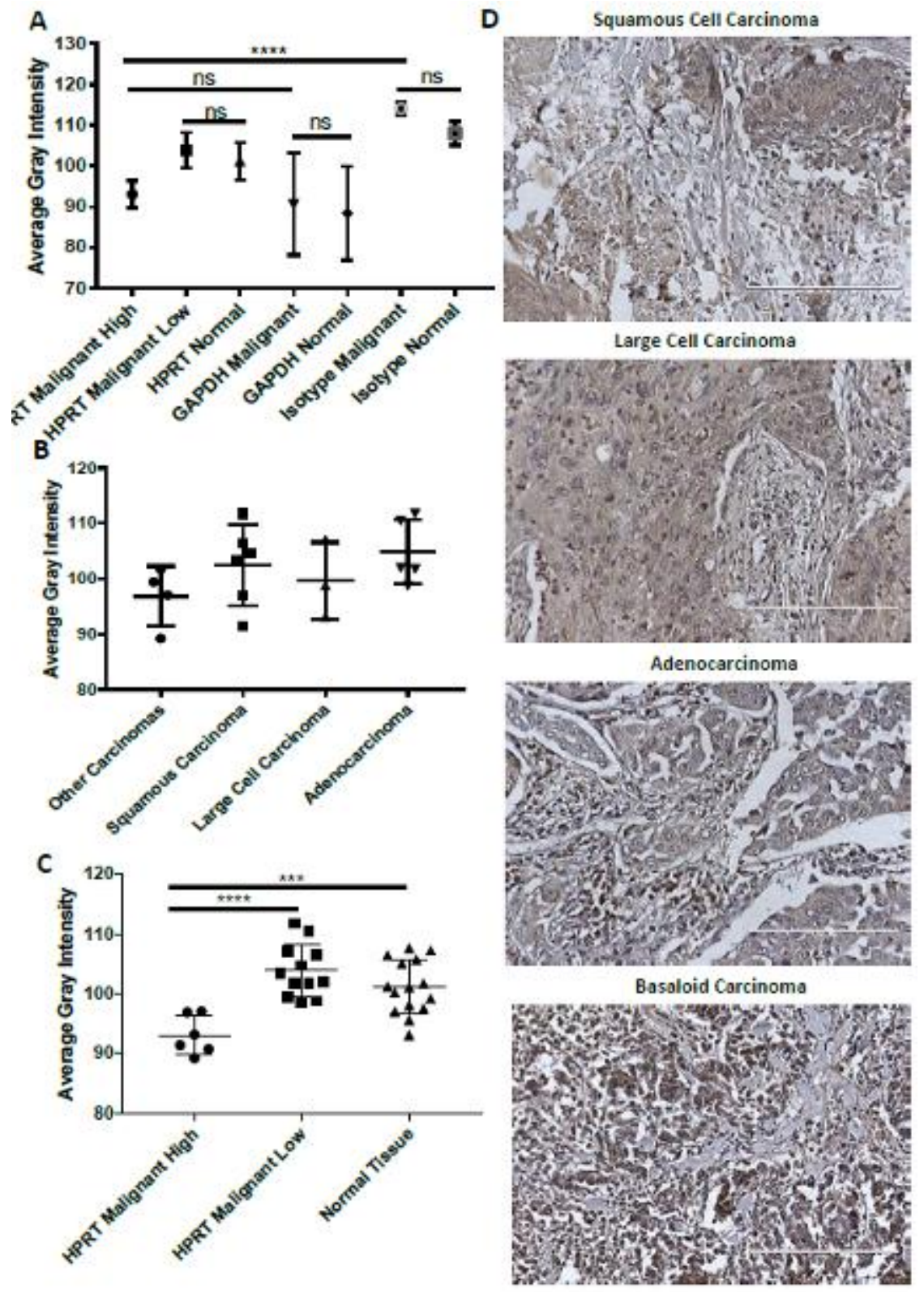

Figure 6. Statistical evaluation of HPRT expression in lung cancer

A) Malignant and normal lung tissue stained with HPRT, GAPDH, and Isotype controls. GAPDH and Isotype samples were not statistically significant between malignant and normal. B) HPRT expression analysis between various tissue types. There was no significance between lung cancer types with regards to HPRT expression. C) There were 6 patients that had elevated HPRT labeled 'HPRT Malignant High' and 12 patients that had insignificant HPRT expression labeled 'HPRT Malignant Low". D) Tissue images of HPRT expression in various lung cancers. 

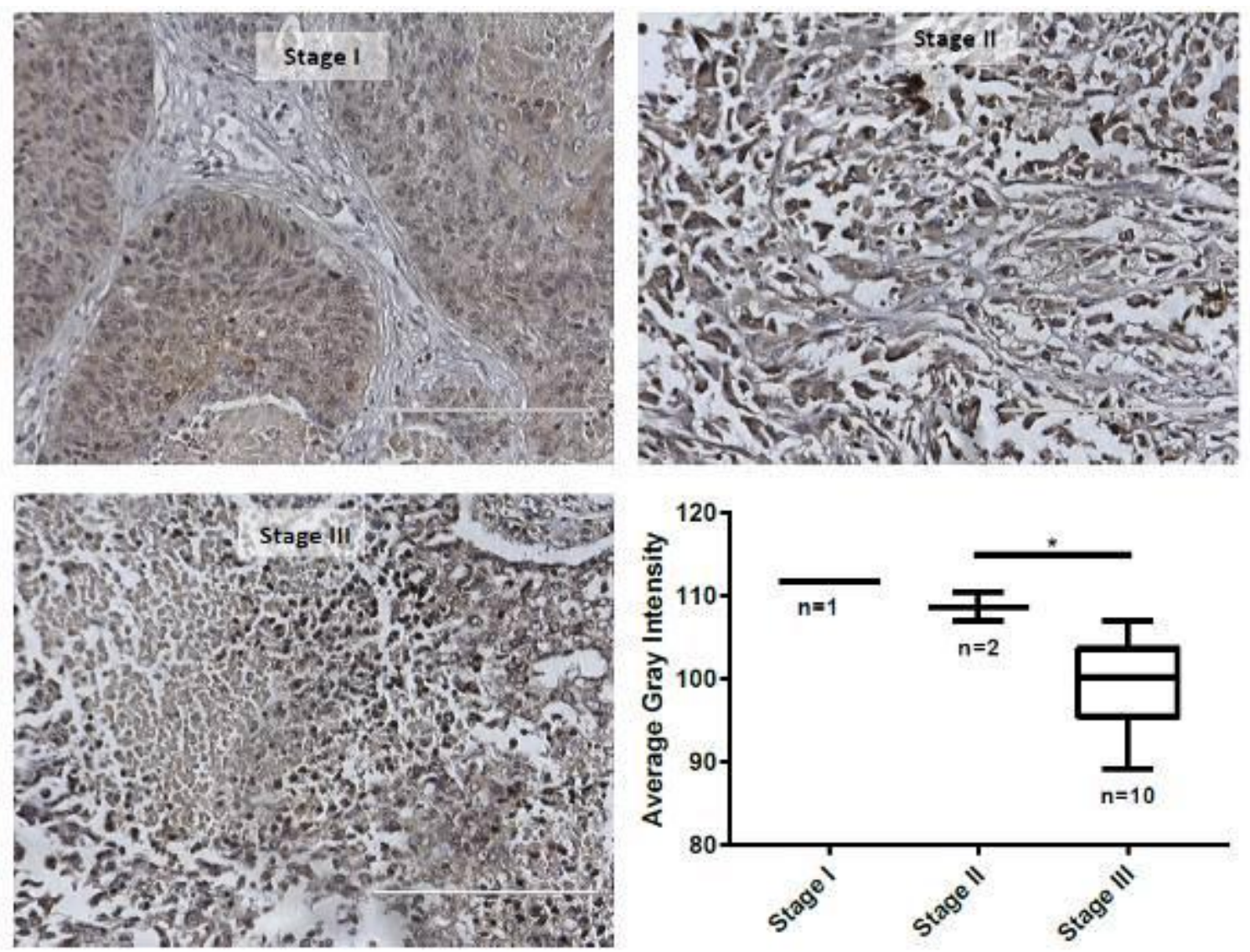

Figure 7. Stage Evaluation of malignant lung tissue

Lung tissue showed significant variations in HPRT expression in relation to stage. Stage I, II, and III tissue are evaluated and imaged to show any variations between tissue intensity.

Lung tissue was the only organ that we observed a stage-dependent increase in HPRT expression. As there was only one stage I tissue to analyze, the significance observed was between stage II and stage III tissue, where there was a significant increase in HPRT expression in stage III tissue $(p=0.05)$. As this pattern was not observed within any other organ, we hypothesize it may be an artifact of the small cohort size and a larger sample size is needed to determine any relevant significance.

\subsection{HPRT Elevation in Metastatic Colon Tumors was Significant When Compared to Primary Tumors}

Within colon cancer tissue, there was a population $(n=18)$ that had a significant upregulation of HPRT when compared to isotype controls (Figure 6A). With an average gray intensity of 92.00, this was significant when compared to both the normal colon tissue controls $(p<0.0001)$ and isotype controls $(p<0.0001)$. Additionally, we also evaluated primary and metastatic colon tumors to determine whether there was any difference in HPRT expression between aggressive, malignant cells that had successfully metastasized and primary tumor cells. We found a similar pattern to other primary tumors evaluated where a subset of patients were 'HPRT High' and a subset of patients who had similar levels to normal controls and were labeled 'HPRT Low' (Figure 8B\&D). We also found that metastatic samples had an overall increase in HPRT expressioncompared to primary tumors $(p=$ 0.014), indicating that metastatic cells may express more HPRT (Figure 8C). 
Table 4. Distribution of HPRT staining in malignant colon tissue and normal colon tissue

\begin{tabular}{lccccc}
\hline Tissue Type & Number of Patients & Grade Range & Age Range & M/F & Overall Gray Intensity \\
\hline Adenocarcinoma & 30 & $1-3$ & $31-79$ & $14 / 16$ & 104.81 \\
\hline Metastatic Adenocarcinoma & 30 & $2-3$ & $30-79$ & $17 / 13$ & 100.37 \\
\hline Tubular Adenoma & 10 & - & $31-69$ & $6 / 4$ & 99.09 \\
\hline Cancer Adjacent Normal Colon Tissue & 20 & - & $32-81$ & $16 / 4$ & 103.01 \\
\hline Normal Colon Tissue & 10 & - & $29-42$ & $10 / 0$ & 105.00 \\
\hline
\end{tabular}

$M / F ;$ Male/Female patients.

A

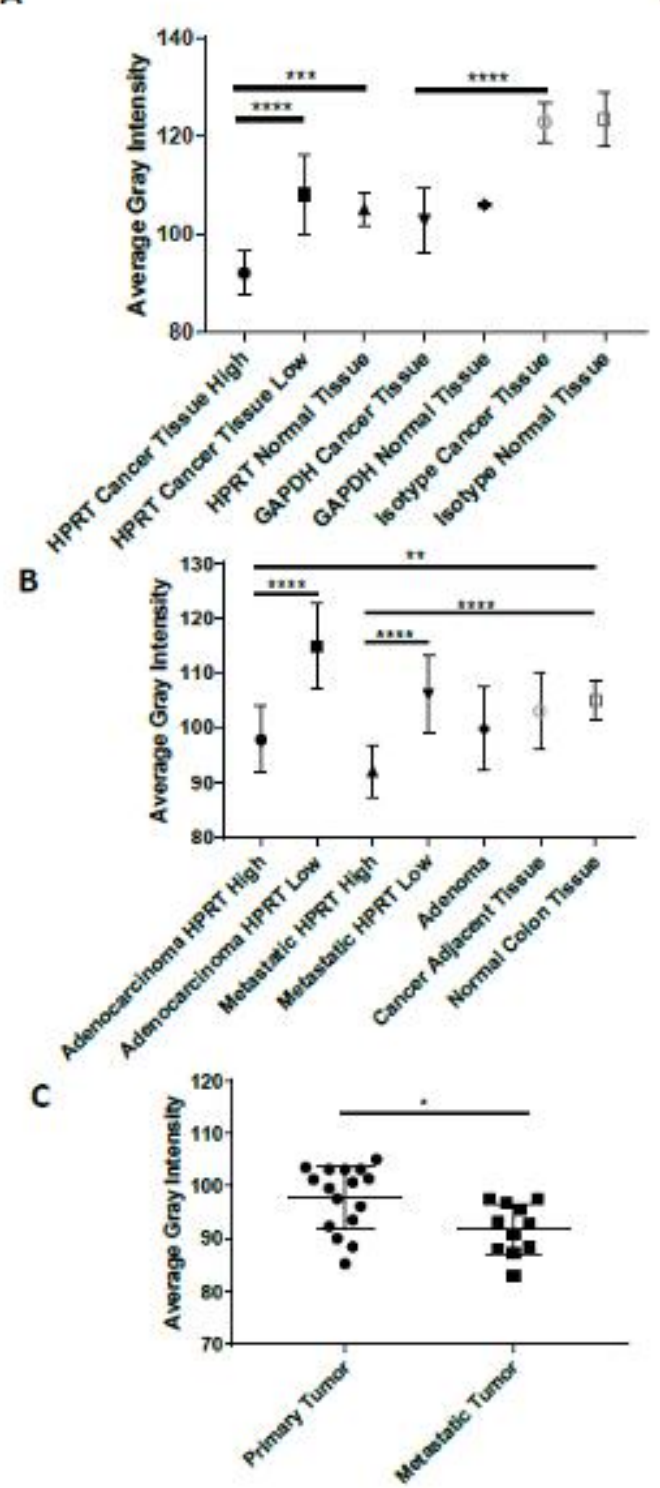

D

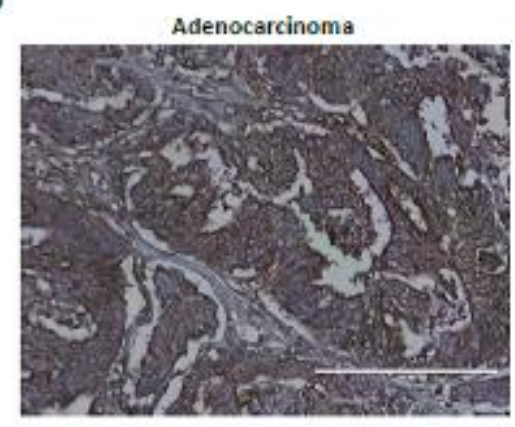

Metastatic Adenocarcinoma from colon

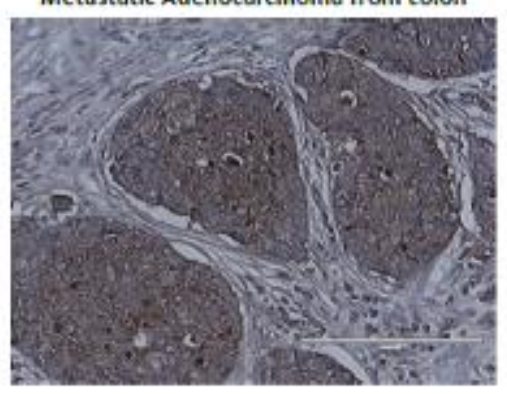

Cancer Adjacent Normal

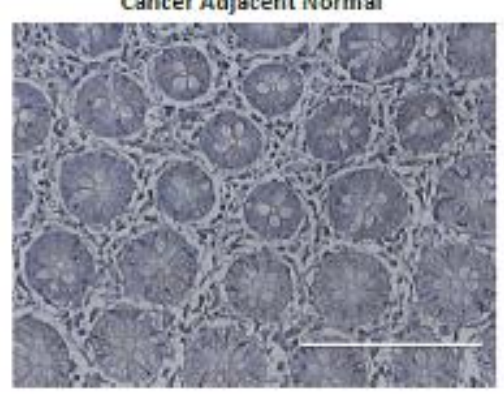

Figure 8. HPRT expression within colon primary tumors, metastatic tumors from the colon, and normal colon tissue

A) Malignant and normal tissue stained with HPRT, GAPDH, and an Isotype antibody. B) HPRT staining within all colon sample tissues. C) 16 primary tumor samples and 11 metastatic tumor samples experienced 'HPRT High' staining. D) Tissue images of HPRT expression in adenocarcinoma, metastatic adenocarcinoma, and normal tissue. 


\subsection{Prostate Cancer Tissue Exhibits Significant HPRT Expression That Is Not Dependent On Stage or Grade}

There was a high percentage $(55 \%)$ of the prostate patient cohort analyzed that was elevated in HPRT when compared to controls (Figure 9). Of the 53 malignant samples analyzzed, 25 individuals experienced significant overexpression of HPRT (Table 5). Following a stage evaluation, we found no statistically relevant correlation between HPRT expression and the cancer stage (Figure 9E). This data, along with data presented in Figure 2, indicates there is a significant population of prostate cancer patients that experience an upregulation of this gene.

Table 5. Distribution of HPRT staining in malignant prostate tissue and normal prostate tissue

\begin{tabular}{lccc}
\hline Tissue Type & Number of Patients & Age Range & Overall Gray Intensity \\
\hline Adenocarcinoma High & 25 & $66-85$ & 110.77 \\
\hline Adenocarcinoma Low & 28 & $60-82$ & 129.56 \\
\hline Hyperplasia & 3 & $63-75$ & 134.20 \\
\hline
\end{tabular}
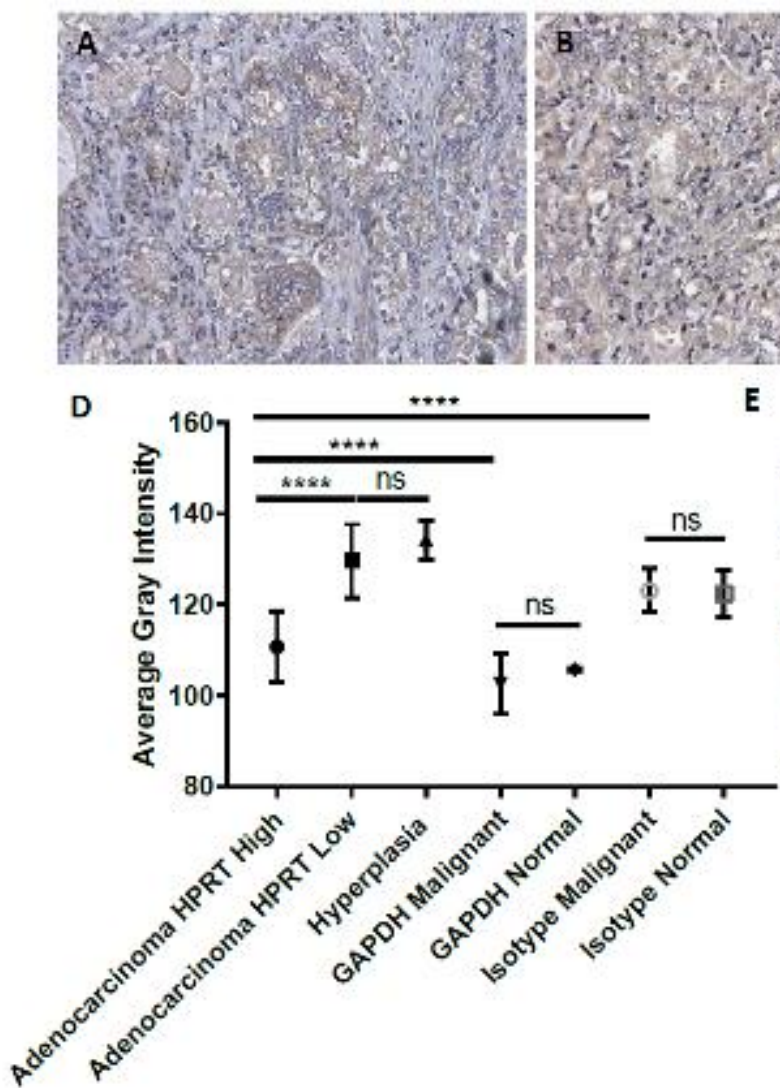

$\mathbf{E}$
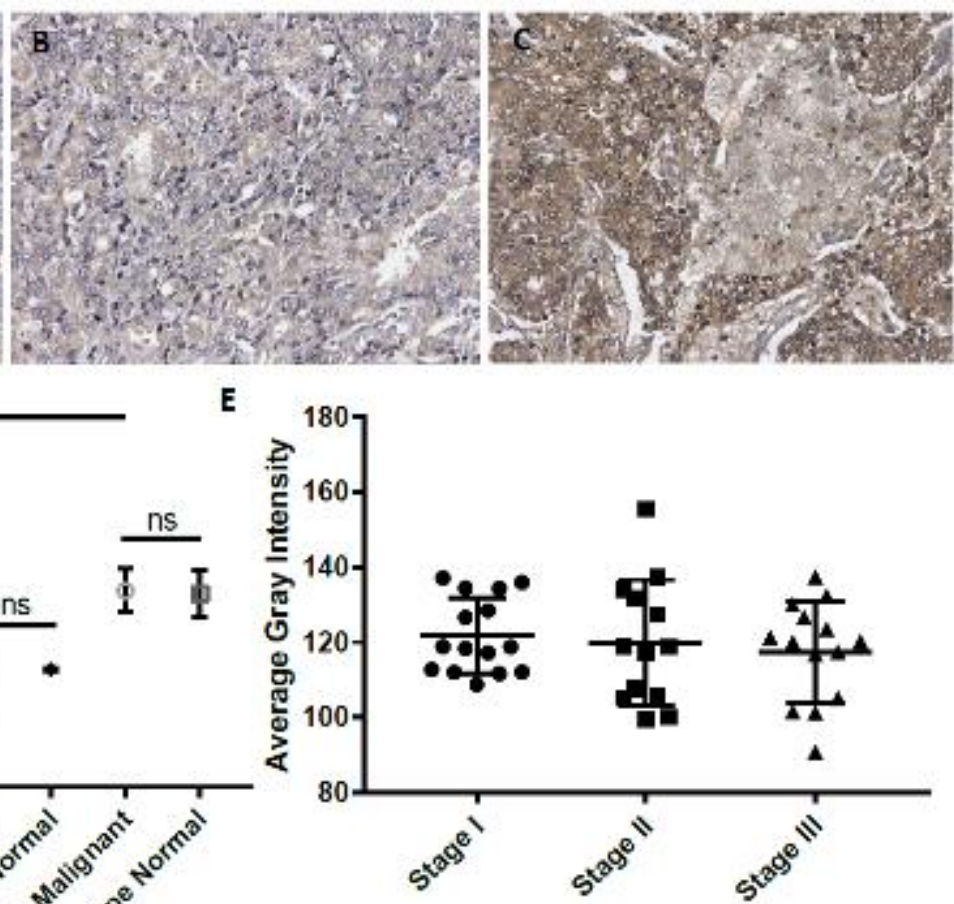

Figure 9. Stage analysis of HPRT expression in prostate cancer stage

Tissue images of A) Stage I; B) Stage II; and C) Stage III Prostate cancer tissue stained with HPRT. D) Staining of prostate tissue with HPRT, GAPDH, and Isotype antibodies. E) Stage evaluation of prostate tissue stained with HPRT shows no statistically relevant differences between cancer stages.

\section{Discussion}

As an enzyme that is significantly upregulated in several malignant tumors, HPRT has the potential to become an additional biomarker for the characterization of several cancers. As Figure 2 indicates, there is significant variability within patientswith regards to their relative expression of HPRT both in normal and malignant tissue with the overall trend showing upregulation of the protein in cancerous tissue. We have evaluated this expression profile in several of the most common malignancies including lung, colon, breast, and prostate cancer with each showing a similar pattern of expression. This leads us to believe this general upregulation within a subset of patients may be a common trend in several cancer types. Further studies will need to be conducted to evaluate 
the expression of this enzyme in other cancer types to determine whether the differential upregulation observed within lung, breast, colon, and prostate cancers is also present within other malignancies. If found as a universal event in all cancer types, HPRT would become useful for cancer characterization and detection.

While there is a basal level of expression due to the housekeeping nature of HPRT, this expression was generally weak in normal tissue (Table 1) and patients who experienced an upregulation had significantly elevated HPRT, orders of magnitude higher than isotype controls. Any upregulation that was observed was also independent of cancer grade or stage with the exception of lung cancer. With this in mind, HPRT could be utilized as an early biomarker because it appears to be upregulated in cancer regardless of stage. This study has expanded the role HPRT currently has as a mutational biomarker to also encompass a possible involvement in cancer development within some patients (Albertini, 2001; Glaab \& Tindall, 1997). These findings are also relevant to recent literature showing that HPRT in some cancer cells may be localized to the surface of the cell, which furthers its potential use as a biomarker for cancer development and potential treatment (Townsend et al., 2017).

As the upregulation of HPRT does not appear to be dependent on cancer stage or grade, the most likely cause of the over-expression is due to mutation. There are several transcription factors that control the expression of HPRT including TP53, NF-KB1, and POU2F1 that we believe may be mutated within these patients that could result in the increase in protein levels within the tumor.

Of interesting note is the use HPRT currently plays as a common endogenous control for several cancer-related studies. Due to its housekeeping nature, HPRT is often utilized as a control for expressional studies and transcriptional analysis in a variety of research techniques (Boonstra et al., 2004; Carver et al., 2009; de Kok et al., 2005; Hillion et al., 2009; Liu, Chen, \& Liu, 2005; Lukasiak et al., 2008; Morimoto-Tomita et al., 2005; Reijm et al., 2011). Yet, the literature is inconsistent when reporting HPRT expression levels within cancer. After comparing various housekeeping genes such as GAPDH, $\beta-2$ microglobulin, 18s ribosomal RNA, etc., some researchers have reported HPRT as the most consistent endogenous control (de Kok et al., 2005). Meanwhile, other researchers have reported HPRT levels to be significantly lower than other controls in cancer tissue (Kheirelseid, Chang, Newell, Kerin, \& Miller, 2010). Finally, other studies have reported HPRT as an unsuitable standard in certain cell types due to varying expression in response to growth factor stimuli(Murphy \& Polak, 2002). Recently, Homey et al. reported the expression of HPRT was detectable in cultured cancer cells, primary tumors, and metastatic tumors, but was found undetectable in normal lung tissue (Homey et al., 2001). This data supports our observations and indicates that HPRT has widely variable expression that would deem it unsuitable as a transcriptional control standard.

\section{Conclusions}

Our results indicate that HPRT has significantly higher expression in malignant tissue when compared to normal controls, and has potential as a biomarker for the characterization of several malignancies including breast, lung, prostate, and colon cancers.

\section{Acknowledgements}

We would like to thank the Simmons Center for Cancer Research for funding this work.

\section{References}

Albertini, R. J. (2001). HPRT mutations in humans: Biomarkers for mechanistic studies. Mutation Research Reviews in Mutation Research, 489(1), 1-16. https://doi.org/10.1016/S1383-5742(01)00064-3

Alegre, M. M., Robison, R. A., \& O'Neill, K. L. O. (2012). Thymidine Kinase 1 Upregulation Is an Early Event in Breast Tumor Formation, https://doi.org/10.1155/2012/575647

Alegre, M. M., Robison, R. A., \& O'Neill, K. L. (2013). Thymidine Kinase 1: A Universal Marker for Cancer. Cancer and Clinical Oncology, 2(1), 159-167. https://doi.org/10.5539/cco.v2n1p159

Alegre, M. M., Weyant, M. J., Bennett, D. T., Yu, J. A., Ramsden, M. K., Elnaggar, A., ... O’Neill, K. L. (2014). Serum detection of thymidine kinase 1 as a means of early detection of lung cancer. Anticancer Research, 34(5), 2145-2152.

American Cancer Society. (2016). Cancer Facts \& Figures 2016. Cancer Facts \& Figures, 1-9. https://doi.org/10.1097/01.NNR.0000289503.22414.79

Boonstra, R., Timmer-Bosscha, H., van Echten-Arends, J., van der Kolk, D. M., van den Berg, a, de Jong, B., ... de Vries, E. G. E. (2004). Mitoxantrone resistance in a small cell lung cancer cell line is associated with ABCA2 upregulation. British Journal of Cancer, 90(12), 2411-2417. https://doi.org/10.1038/sj.bjc.6601863 
Branda, R. F., O’Neill, J. P., Jacobson-Kram, D., \& Albertini, R. J. (1992). Factors influencing mutation at the hprt locus in T-lymphocytes: studies in normal women and women with benign and malignant breast masses. Environ Mol Mutagen, 19(4), 274-281.

Carver, B. S., Tran, J., Gopalan, A., Chen, Z., Shaikh, S., Carracedo, A., ... Pandolfi, P. P. (2009). Aberrant ERG expression cooperates with loss of PTEN to promote cancer progression in the prostate. Nature Genetics, 4l(5), 619-24. https://doi.org/10.1038/ng.370

Chang, Y.-J., Tseng, C.-Y., Lin, P.-Y., Chuang, Y.-C., \& Chao, M.-W. (2017). Acute exposure to DEHP metabolite, MEHP cause genotoxicity, mutagenesis and carcinogenicity in mammalian Chinese hamster ovary cells. Carcinogenesis, 38(3), 336-345. https://doi.org/10.1093/carcin/bgx009

Cheng, T., Christiani, D. C., Liber, H. L., Wain, J. C., Xu, X., Wiencke, J. K., \& Kelsey, K. T. (1995). Mutant frequency at the hprt locus in human lymphocytes in a case-control study of lung cancer, 332.

de Kok, J. B., Roelofs, R. W., Giesendorf, B. a, Pennings, J. L., Waas, E. T., Feuth, T., ... Span, P. N. (2005). Normalization of gene expression measurements in tumor tissues: comparison of 13 endogenous control genes. Laboratory Investigation; a Journal of Technical Methods and Pathology, 85(1), 154-159. https://doi.org/10.1038/labinvest.3700208

Duthie, S. J., Ross, M., \& Collins, A. R. (1995). The influence of smoking and diet on the hypoxanthine phosphoribosyltransferase (hprt) mutant frequency in circulating $\mathrm{T}$ lymphocytes from a normal human population. Mutation Research - Fundamental and Molecular Mechanisms of Mutagenesis, 331(1), 55-64. https://doi.org/10.1016/0027-5107(95)00051-J

Glaab, W., \& Tindall, K. (1997). Mutation rate at the hprt locus in human cancer cell lines with specific mismatch repair-gene defects. Carcinogenesis, 18(1), 1-8.

Gobrecht, J., McDyre, C., Comotto, J., \& Reynolds, M. (2017). Induction of cytotoxic and genotoxic damage following exposure of V79 cells to cadmium chloride. Mutation Research/Genetic Toxicology and Environmental Mutagenesis, 816-817, 12-17. https://doi.org/10.1016/j.mrgentox.2017.03.001

Grist, S. a, McCarron, M., Kutlaca, a, Turner, D. R., \& Morley, a a. (1992). In vivo human somatic mutation: frequency and spectrum with age. Mutation Research, 266(2), 189-196. https://doi.org/10.1016/0027-5107(92)90186-6

Hakoda, M., Akiyama, M., Kyoizumi, S., \& Awa, A. A. (1988). Increased somatic cell mutant frequency in atomic bomb survivors, 201, 39-48.

He, Q., Fornander, T., Johansson, H., Johansson, U., Hu, G. Z., Rutqvist, L. E., \& Skog, S. (2006). Thymidine kinase 1 in serum predicts increased risk of distant or loco-regional recurrence following surgery in patients with early breast cancer. Anticancer Research, 26(6C), 4753-4759.

Hillion, J., Wood, L. J., Mukherjee, M., Bhattacharya, R., Di Cello, F., Kowalski, J., ... Resar, L. M. S. (2009). Upregulation of MMP-2 by HMGA1 promotes transformation in undifferentiated, large-cell lung cancer. Molecular Cancer Research : MCR, 7(11), 1803-1812. https://doi.org/10.1158/1541-7786.MCR-08-0336

Hirota, H., Kubota, M., Hashimoto, H., Adachi, S., \& Mikawa, H. (1993). Analysis of hprt gene mutation following anti-cancer treatment in pediatric patients with acute leukemia Haruyo Hirota a , Masaru Kubota a , Hisako Hashimoto a , Souichi Adachi a , Kousaku Matsubara a , Katsuji Kuwakado a, Yuichi Akiyama a, Tsutomu Tsut, 319, 113-120.

Homey, B., Soto, H., Ge, N., Catron, D., Buchanan, M. E., Mcclanahan, T., ... Vera, E. (2001). Involvement of chemokine receptors in breast cancer metastasis. Nature, 410(6824), 50-56.

Hou, S. M., Yang, K., Nyberg, F., Hemminki, K., Pershagen, G., \& Lambert, B. (1999). Hprt mutant frequency and aromatic DNA adduct level in non-smoking and smoking lung cancer patients and population controls. Carcinogenesis, 20(3), 437-444.

Jagarlamudi, K. K., Hansson, L. O., \& Eriksson, S. (2015). Breast and prostate cancer patients differ significantly in their serum Thymidine kinase 1 ( TK1 ) specific activities compared with those hematological malignancies and blood donors: implications of using serum TK1 as a biomarker, 1-13. https://doi.org/10.1186/s12885-015-1073-8

Keough, D. T., Brereton, I. M., De Jersey, J., \& Guddat, L. W. (2005). The crystal structure of free human hypoxanthine-guanine phosphoribosyltransferase reveals extensive conformational plasticity throughout the catalytic cycle. Journal of Molecular Biology, 351(1), 170-181. https://doi.org/10.1016/j.jmb.2005.05.061 
Kheirelseid, E. A. H., Chang, K. H., Newell, J., Kerin, M. J., \& Miller, N. (2010). Identification of endogenous control genes for normalisation of real-time quantitative PCR data in colorectal cancer. BMC Molecular Biology, 11, 12. https://doi.org/10.1186/1471-2199-11-12

Liu, D. W., Chen, S. T., \& Liu, H. P. (2005). Choice of endogenous control for gene expression in nonsmall cell lung cancer. The European Respiratory Journal: Official Journal of the European Society for Clinical Respiratory Physiology, 26(6), 1002-1008. https://doi.org/10.1183/09031936.05.00050205

Lukasiak, S., Schiller, C., Oehlschlaeger, P., Schmidtke, G., Krause, P., Legler, D. F., ... Groettrup, M. (2008). Proinflammatory cytokines cause FAT10 upregulation in cancers of liver and colon. Oncogene, 27(46), 6068-6074. https://doi.org/10.1038/onc.2008.201

Monnat, R. J., Chiaverotti, T. a, Hackmann, a F., \& Maresh, G. a. (1992). Molecular structure and genetic stability of human hypoxanthine phosphoribosyltransferase (HPRT) gene duplications. Genomics, 13(3), 788-96.

Morimoto-Tomita, M., Uchimura, K., Bistrup, A., Lum, D. H., Egeblad, M., Boudreau, N., ... Rosen, S. D. (2005). Sulf-2, a proangiogenic heparan sulfate endosulfatase, is upregulated in breast cancer. Neoplasia, 7(11), 1001-1010. https://doi.org/10.1593/neo.05496

Murphy, C. L., \& Polak, J. M. (2002). Differentiating embryonic stem cells: GAPDH, but neither HPRT nor beta-tubulin is suitable as an internal standard for measuring RNA levels. Tissue Engineering, 8(4), 551-559. https://doi.org/10.1089/107632702760240472

O’Neill, J. P., Nicklas, J. A., Hunter, T. C., Batson, O. B., Allegretta, M., Falta, M. T., ... Albertini, R. J. (1994). The effect of T-lymphocyte "clonality" on the calculated hprt mutation frequency occurring in vivo in humans. Mutation Research/Environmental Mutagenesis and Related Subjects, 313(2-3), 215-225. https://doi.org/10.1016/0165-1161(94)90052-3

Reijm, E. A., Jansen, M. P. H. M., Ruigrok-Ritstier, K., Van Staveren, I. L., Look, M. P., Van Gelder, M. E. M., ... Berns, E. M. J. J. (2011). Decreased expression of EZH2 is associated with upregulation of ER and favorable outcome to tamoxifen in advanced breast cancer. Breast Cancer Research and Treatment, 125(2), 387-394. https://doi.org/10.1007/s10549-010-0836-9

Robinson, D. R., Albertini, R. J., Neill, O., Finette, B., Sala-trepat, M., Moustacchi, E., ... Cole, J. (1994). An analysis of in vivo hprt mutant frequency in circulating T-lymphocytes in the normal human population : a comparison of four datasets, 313, 227-247.

Sawada, M., Kubota, M., Lin, Y. W., Watanabe, K., Koishi, S., Usami, I., ... Furusho, K. (1998). Prospective study of mutant frequencies at the hprt and T-cell receptor gene loci in pediatric cancer patients during chemotherapy. Cancer Epidemiol Biomarkers Prev, 7(8), 711-717.

Siegel, R. L., Miller, K. D., \& Jemal, A. (2016). Cancer statistics. CA Cancer J Clin, 66(1), 7-30. https://doi.org/10.3322/caac.21332.

Siegel, R., Miller, K., \& Jemal, A. (2015). Cancer statistics , 2015 . CA Cancer J Clin, 65(1), 29. https://doi.org/10.3322/caac.21254.

Stout, J. T., \& Caskey, C. T. (1985). Hprt: gene structure, expression, and mutation.

Tates, A. D., Dam, F. J. Van, Natarajan, A. T., Zwinderman, A. H., \& Osanto, S. (1994). Frequencies of HPRT mutants and micronuclei in lymphocytes of cancer patients under chemotherapy: a prospective study, 307, 293-306.

Townsend, M. H., Anderson, M. D., Weagel, E. G., Velazquez, E. J., Weber, K. S., Robison, R. A., \& O'Neill, K. L. (2017). Non-small-cell lung cancer cell lines A549 and NCI-H460 express hypoxanthine guanine phosphoribosyltransferase on the plasma membrane. OncoTargets and Therapy, 10, 1921-1932. https://doi.org/10.2147/OTT.S128416

Vacek, P. M., \& Albertini, R. J. (1993). M e a s u r e m e n t of H P R T mutant frequencies in T-lymphocytes from healthy human populations R. F. Branda a , L . M . Sullivan a j . p . O a $€^{\mathrm{TM}}$ Neill a M . T . Falta a , J . A . Nicklas a B . Hirsch c , 285, 267-279.

Welcome to Python.org. (n.d.). Retrieved July 19, 2017, from https://www.python.org/

Wilson, J. M., Tarr, G. E., \& Kelley, W. N. (1983). Human hypoxanthine (guanine) phosphoribosyltransferase: an amino acid substitution in a mutant form of the enzyme isolated from a patient with gout. Proceedings of the National Academy of Sciences of the United States of America, 80(3), 870-3. 
https://doi.org/10.1073/pnas.80.3.870

Worldwide cancer statistics | Cancer Research UK. Retrieved July 18, 2017, from

http://www.cancerresearchuk.org/health-professional/cancer-statistics/worldwide-cancer

\section{Copyrights}

Copyright for this article is retained by the author(s), with first publication rights granted to the journal.

This is an open-access article distributed under the terms and conditions of the Creative Commons Attribution license (http://creativecommons.org/licenses/by/4.0/). 